\title{
Myocardial tissue characterisation with late gadolinium enhancement in rheumatoid arthritis, systemic lupus erythematosus and systemic sclerosis
}

\author{
Ntobeko A Ntusi ${ }^{1 *}$, Jane M Francis ${ }^{1}$, Paul M Matthews², Paul B Wordsworth ${ }^{3}$, Stefan Neubauer ${ }^{1}$, \\ Theodoros Karamitsos ${ }^{1}$
}

From 16th Annual SCMR Scientific Sessions

San Francisco, CA, USA. 31 January - 3 February 2013

\section{Background}

Rheumatoid arthritis (RA), systemic lupus erythematosus (SLE) and systemic sclerosis (SSc) commonly involve the cardiovascular system, and are associated with high morbidity and mortality. Mechanisms of cardiovascular disease (CVD) involvement in these clinical entities are not fully understood. Furthermore, little is known about myocardial structure and function in these inflammatory arthropathies. Late gadolinium enhancement (LGE) cardiovascular magnetic resonance (CMR) imaging is a tool for noninvasive evaluation of myocardial fibrosis that has the advantage over other imaging techniques of being able to directly visualise both ischaemic and nonischaemic patterns of injury, and has prognostic significance. The purpose of this study was to assess the frequency and pattern of LGE in RA, SLE and SSc patients without any known CVD using CMR and to determine its relation to disease duration, vascular function (aortic distensibility; pulse wave velocity) and left ventricular
(LV) systolic function (LV ejection fraction; mid short axis circumferential systolic strain).

\section{Methods}

59 RA patients (42 female, mean age $53 \pm 12$ ), 29 SLE patients ( 28 female, mean age $42 \pm 10$ ), 18 SSc patients (17 female, mean age $55 \pm 10$ ), 45 normal controls (38 female, mean age $44 \pm 12$ ), and 14 controls with cardiovascular risk factors [CVRFs] (11 female, mean age $52 \pm 8$ ) underwent CMR at 1.5 Tesla. All patients with previously known CVD were excluded. Biventricular volumes and function, presence and pattern of LGE, myocardial strain and vascular function were assessed by CMR.

\section{Results}

LGE was found to occur in $46 \%$ of RA, 31\% of SLE and $50 \%$ of SSc patients compared to $9 \%$ of normal controls and $21 \%$ of controls with CVRFs $(\mathrm{p}<0.001)$. The patterns of LGE are presented in Table 1. Presence of LGE correlated

Table 1 Frequency and patterns of LGE in RA, SLE and SSc patients and in normal controls and controls with CVRFs.

\begin{tabular}{|c|c|c|c|c|c|c|}
\hline & $\begin{array}{l}\text { Normal } \\
\text { controls } \\
\mathrm{N}=45\end{array}$ & $\begin{array}{l}\text { Controls } \\
\text { with CVRFs } \\
\mathrm{N}=14\end{array}$ & $\mathrm{RA} N=59$ & SLE N=29 & $\mathrm{SSC} \mathrm{N}=18$ & P Value \\
\hline Frequency of LGE & $4(8.9)$ & $3(21.4)$ & $27(45.8)$ & $9(31.0)$ & $9(50.0)$ & $<0.001$ \\
\hline $\begin{array}{c}\text { Pattern of LGE Basal inferolateral LGE Lateral wall } \\
\text { midwall/subepicardial LGE Septal midwall LGE Myocardial } \\
\text { infarction }\end{array}$ & $\begin{array}{l}1(2.2) 1 \\
(2.2) 2(4.4) \\
0(0)\end{array}$ & $\begin{array}{c}2(14.3) 0(0) \\
1(7.1) 0(0)\end{array}$ & $\begin{aligned} 16 & (27.1) 5 \\
(8.5) & 3(5.1) 3 \\
& (5.1)\end{aligned}$ & $\begin{array}{l}5(17.2) 2 \\
(6.9) 0(0) 2 \\
(6.9)\end{array}$ & $\begin{array}{c}4(22.2) 5 \\
(27.8) 0(0) 0 \\
(0)\end{array}$ & $\begin{array}{l}<0.001<0.001 \\
<0.001<0.001\end{array}$ \\
\hline
\end{tabular}

${ }^{1}$ OCMR, Cardiovascular Medicine, University of Oxford, Oxford, UK

Full list of author information is available at the end of the article

(c) 2013 Ntusi et al; licensee BioMed Central Ltd. This is an Open Access article distributed under the terms of the Creative Commons 


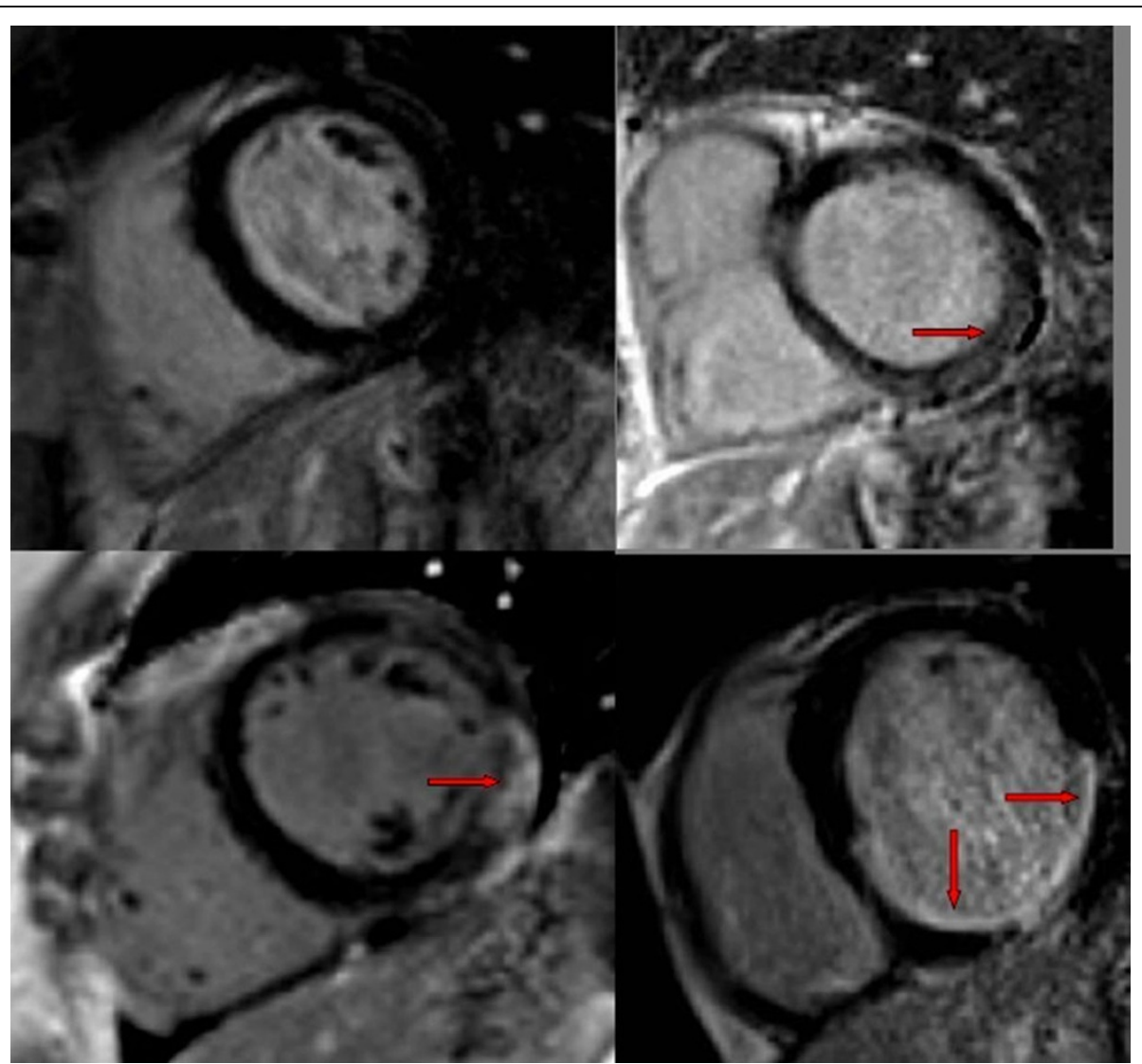

Figure 1 Different patterns of LGE in patients with inflammatory arthropathies (Top left: control with no LGE; top right: SSC patient with basal inferolateral wall LGE; bottom left: SLE patient with lateral midwall/subepicardial LGE; bottom right: RA patient with myocardial infarction (subendocardial LGE).

with disease duration $(\mathrm{Rs}=0.33 ; \mathrm{p}<0.001)$, myocardial strain $(\mathrm{Rs}=0.29 ; \mathrm{p}<0.001)$, and total aortic pulse wave velocity $(\mathrm{Rs}=0.72 ; \mathrm{p}<0.001)$.

\section{Conclusions}

CMR demonstrates an increased burden of both ischaemic and non-ischaemic fibrosis in patients with inflammatory systemic diseases with no known CVD. Increased myocardial fibrosis may contribute to the poor cardiovascular outcomes in this group of patients. LGE in RA, SLE and SSc correlates with increasing disease duration, impaired myocardial strain, and increased pulse wave velocity.

\section{Funding}

NN is funded by the Discovery Foundation and Nuffield Trust. SN acknowledges support from the British Heart Foundation Centre of Research Excellence, Oxford. The research was funded through an investigator-led grant from GSK.

\section{Author details}

'OCMR, Cardiovascular Medicine, University of Oxford, Oxford, UK. ${ }^{2}$ GSK Clinical Imaging Centre, Imperial College, London, UK. ${ }^{3}$ Bortnar Institute and Nuffield Department of Orthopaedics, Rheumatology and Musculoskeletal Sciences, University of Oxford, Oxford, UK.

Published: 30 January 2013

\section{doi:10.1186/1532-429X-15-S1-047}

Cite this article as: Ntusi et al:: Myocardial tissue characterisation with late gadolinium enhancement in rheumatoid arthritis, systemic lupus erythematosus and systemic sclerosis. Journal of Cardiovascular Magnetic Resonance 2013 15(Suppl 1):047. 